EPJ Web of Conferences 88, 00019 (2015)

DOI: $10.1051 /$ epjconf/ 20158800019

(C) Owned by the authors, published by EDP Sciences - SIF, 2015

\title{
Studying heavy-ion collisions with FAUST-QTS
}

P. Cammarata ${ }^{1,2}$, M. B. Chapman ${ }^{1,3}$, G. A. Souliotis $^{1,4}$, L. Bakhtiari ${ }^{1,3}$, S. Behling ${ }^{1,2}$, G. Bonasera ${ }^{1,3}$, L. A. Heilborn ${ }^{1,2}$, J. Mabiala ${ }^{1}$, A. B. McIntosh ${ }^{1}$, L. W. MaY ${ }^{1,2}$, A. Raphelt ${ }^{1,2}$, M. D. Youngs $^{1}$, A. Zarrella ${ }^{1,2}$ and S. J. Yennello ${ }^{1,2}$

${ }^{1}$ Cyclotron Institute, Texas A\&M University, College Station, TX, USA

${ }^{2}$ Chemistry Department, Texas A\&M University, College Station, TX, USA

${ }^{3}$ Physics Department, Texas A\&M University, College Station, TX, USA

${ }^{4}$ Chemistry Deparment, National and Kapodistrian University of Athens and Hellenic Institute of Nuclear Physics, Athens 15771, Greece

\begin{abstract}
Heavy-ion collisions at lower energies provide a rich environment for investigating reaction dynamics. Recent theory has suggested a sensitivity to the symmetry energy and the equation of state via deformations of the reaction system and ternary breaking of the deformed reaction partners into three heavy fragments. A new detection system has been commissioned at Texas A\&M University in an attempt to investigate some of the observables sensitive to the nuclear equation of state.
\end{abstract}

\section{Introduction}

Heavy-ion collisions below the Fermi energy (10 -15A MeV) provide a rich environment to study reaction dynamics as there are a number of competing reactions mechanisms at play. This competition between dynamical emission, pre-equilibrium emission and statistical decay of reaction products results in the entanglement of a number of underlying processes. The 
scope of the initial commissioning phase of this campaign is focused on examining the dynamical break-up of the projectile- and target-like fragments from heavy-ion collisions. Specifically, we are interested in investigating the reaction mechanism competition involved to produce heavy $(\mathrm{Z} \geq 3$ ) 3body breaking of the reacting system at lower-intermediate beam energies ( $\sim 15 \mathrm{MeV} /$ nucleon). In this specific case, it has been proposed that the isospin content, alignment and velocity distributions of the excited primary fragments, shape deformations of the heaviest fragments, and observables associated with the emission of fragments from the low-density neck region (multiplicity, iso-spin content, angular alignment, etc) produced in mid-peripheral heavy ion collisions represent observables sensitive to the nEoS [1-5]. Although there are a number of other detectors that are capable of measuring light particles and/or heavy, slower particles near the beam axis [6-9], this particular experiment attempts to measure a significant fraction of the observables from both the heavy, projectile-like fragments (PLFs) as well as the emitted intermediate mass fragments (IMFs) at lower energies. Data collected for the purposes of this experiment were from reactions of $15 \mathrm{MeV} /$ nucleon ${ }^{136} \mathrm{Xe}+{ }^{64} \mathrm{Ni}$ using the K500 super-conducting cyclotron at Texas A\&M University.

\section{Experimental design and considerations}

The FAUST array [10] has been coupled to a large quadrupole triplet spectrometer (QTS) previously used for radioactive ion beam (RIB)/facility upgrade experiments with BIGSOL $[9,11-14]$. FAUST-QTS was designed to collect Time-of-Flight (ToF) mass measurements in FAUST and QTS for heavy PLFs as well as $\triangle \mathrm{E}-\mathrm{E}$ measurements in FAUST for good isotopic resolution of LCPs and IMFs $[10,15]$. The ToF mass identification in FAUST is achieved via newly designed integrated charge sensitive, timing pickoff (CS/TPO) preamplifier upgrade to FAUST combined with a thin film, fast plastic time-zero detector up-stream of FAUST. The ToF mass measurements in the QTS were made using Parallel Plate Avalanche Counters (PPACs) [16] for time coupled with a Si semiconductor detector at $\theta=0^{\circ}$ for energy. In forward-focused intermediate energy nuclear reactions, this allows for collection of the resultant PLF in coincidence with emitted IMFs.

FAUST is composed of $68 \mathrm{Si}-\mathrm{CsI}(\mathrm{Tl}) \Delta \mathrm{E}-\mathrm{E}$ telescopes arranged into five concentric Rings $[10,15]$. In the current configuration of FAUST-QTS, the external Zepto systems preamplifiers [17] were replaced with new chargesensitive timing pick-off (CS-TPO) preamplifiers and motherboards from 
RIS-Corp [18] for Rings A-D silicon detectors. The new CS-TPO preamplifiers occupy a slightly larger footprint than the Zepto systems preamplifiers. In the lab, we were able to achieve a time resolution of up to $\sim 150 \mathrm{ps}$ full-width at half-maximum (FWHM). This ideal resolution was most likely possible because of a small angular coverage of the detector due to distance from the source combined with low background noise and single isotope analysis. However, in the actual experiment, we were able to achieve a maximum timing resolution of $\sim 400$ ps maximum resolution FWHM. The fast plastic scintillator used for the stop signal in the ToF measurements in FAUST, was a $10 \mu \mathrm{m}$ thick, BC-408 fast scintillating plastic. The fast plastic was mounted inside a custom fabricated housing upstream of the FAUST array for the experiment. ToF mass measurements in the QTS were made by measuring the ToF between two PPACs in conjunction with the collection of the full energy signal from a $1000 \mu \mathrm{m}$ silicon detector (MSQ-25, Micron Semiconductor, Lancing, UK). The Quadrupole Triplet Spectrometer (QTS) consists of three large bore (8 inch inner-diameter) quadrupole magnets focusing in the $\mathrm{X}-, \mathrm{Y}$-, and then $\mathrm{X}$-direction (respectively) relative to the beam axis (Z-axis) in the laboratory frame of reference. The spectrometer also consists of two customizable detection chambers, one before and one after the magnets, to allow ToF mass particle identification (PID) and/or $\Delta \mathrm{E}-\mathrm{E}$ isotopic identification depending on the energies of the particles of interest. For this experiment we employed the ToF mass PID. This was accomplished using a Parallel Plate Avalanche (PPAC) detector before and after the quadrupole triplet, for accurate time of flight measurement, and a $5 \mathrm{~cm} \times 5 \mathrm{~cm}, 1000 \mu \mathrm{m}$ thick, quadrant segmented silicon semiconductor detector for accurate particle energy measurement. The total particle flight path length was $657 \mathrm{~cm}$ (linear) through the FAUST-QTS.

\section{Simulations}

An important consideration of using a spectrometric device is understanding the physics that affects particle transport. This is important in order to efficiently detect the particles of interest. The particles' mass, charge state, angle of entrance into the spectrometer, and momentum are all important considerations that must be carefully taken into account when calculating charged particle transport flight paths. Several rounds of simulations have been examined for number of probable cases of transport through the QTS using RAYTRACE [19] and COSY-Infinity [20]. This was done in order to examine the theoretical, optical transport considerations required to con- 


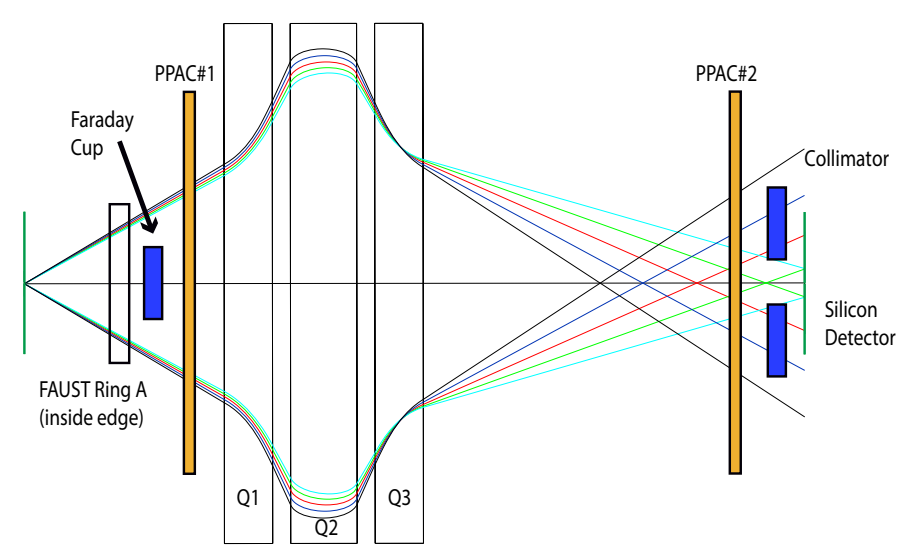

Figure 1: COSY-Infinity simulations showing the YZ-plane flight path of ${ }^{136} \mathrm{Xe}$ at 15 $\mathrm{MeV} /$ nucleon at $\theta=1.5^{\circ}$. The five, discrete magnetic rigidities are centered around a $B \rho=1.45 \mathrm{Tm}$ for the QTS magnets. Red $= \pm 0 \% B \rho$, Light Blue $=+5 \% B \rho$, Dark Blue $=-5 \% B \rho$, Green $=+10 \% B \rho$, and Black $=-10 \% B \rho$.

duct the proposed experiment. Simulations via COSY-Infinity illustrate the effects of $B \rho$ on focusing particles through the triplet, as shown in Figure 1. Figure 1 illustrates several values of $B \rho$ for a single theta value of $\theta=1.5^{\circ}$. As the value of theta increases (up to a maximum of $\theta=2.3^{\circ}$ ) so do the aberrations in the focal image. This is particularly true for the YZ-plane where it can be seen that even at $\theta=1.5^{\circ}$ the flight paths of the particles are nearing the edge of the triplet aperture in the $2^{\text {nd }}$ quadrupole magnet (Q2). The aberration in the XZ-plane is less than that of the YZ-plane simply because Q1 focuses in the X-direction, thereby initially defocusing in the $\mathrm{Y}$-direction.

\section{Experimental results}

The damped, PLF-like events of interest in the QTS coincident with an IMF in FAUST, are shown in the energy versus time plot on Figure 2. The events were required to pass an additional cut requiring the mass of the PLF to be between $100 \leq A_{P L F} \leq 195$. The lines of constant mass were calculated relativistically. In the QTS, the energy and time calibration were completed using a combination of elastically scattered beam particles and a pulser.

The mean free path through the spectrometer was used in the calculation of the flight time. Although mass unit resolution was not possible, the calibrated mass lines (with error bars) show that there is about a 2-3 mass 


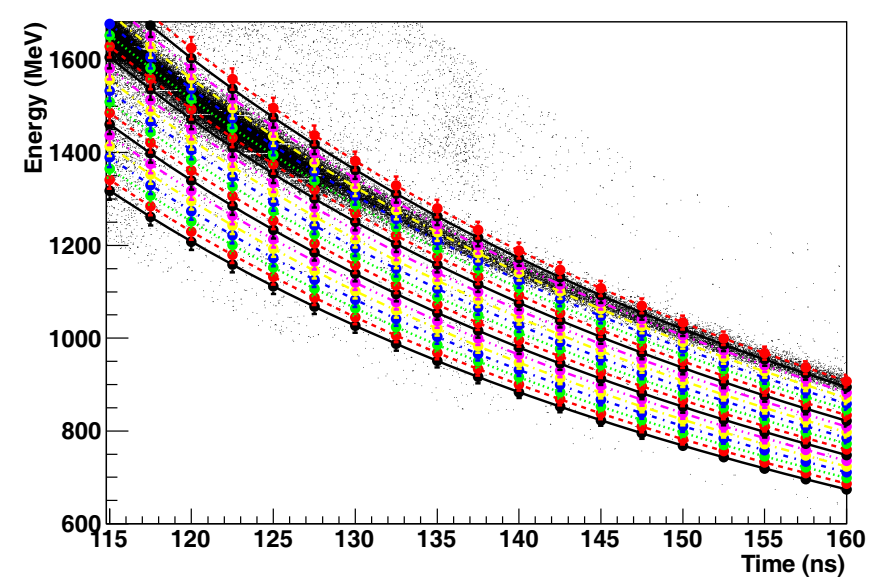

Figure 2: Energy versus time plot for the PLF-like events of interest. The lines of constant mass shown represent a range of all even masses from $A=110$ (lowest back, solid line) to $A=148$ (upper red, dashed lines). Error bars are shown both for energy and time. The error in the calibration represents a mass error of 2-3 mass units.

unit resolution. In the linearized mass spectra it was seen that the magnetic rigidity of the tuned triplet covered a range of masses below and above the mass of the projectile.

\section{Summary}

A new beam line has been built for the study of heavy-ion reaction products below the Fermi energy. The FAUST-QTS should offer the ability to study reaction products near the beam axis via zero-degree, quadrupole spectrometer combined with a forward array to measure the off beam axis LCPs and IMFs. The detector arrangement has met the minimum design criteria and has been recently used to study the fragmentation of ${ }^{136} \mathrm{Xe},{ }^{124} \mathrm{Sn}$, and ${ }^{124} \mathrm{Xe}$ projectiles on ${ }^{64} \mathrm{Ni}$ and ${ }^{58} \mathrm{Ni}$ targets. Work is currently underway to improve the energy and time (and thereby the mass) resolution of the QTS. FAUST currently offers good isotopic resolution via $\Delta E-E$ and work is underway to study pulse shape digitization and analysis via the $\mathrm{CsI}(\mathrm{Tl})$ photodiodes [21]. Further experiments are in the development stage to utilize FAUST-QTS for temperature and particle correlation experiments. 


\section{Acknowledgments}

We are grateful to the Texas A\&M Super-Computing facility and the Texas A\&M Chemistry Department's Laboratory for Molecular Simulations for the generous grant of over 10 million High Performance Computing (HPC) processor hours (combined). This work supported by grants from the Robert A. Welch Foundation Grant No. A-1266, the U. S. Department of Energy under Grant No. DE-FG03-93ER-40773, and National Science Foundation Grant Nos. CHE-0541587 and PHY-1102511.

\section{References}

[1] Colonna M., J. Phys. Conf. Ser. 168012006 (2009).

[2] Rizzo C. et al., Phys. Rev. C 8301460 (2011).

[3] Di Toro M. et al., Nucl. Phys. A 787585 (2007).

[4] Shvedov L., Colonna M. and Di Toro M., Phys. Rev. C 81054605 (2010).

[5] Giuliani G., Zheng H. and Bonasera A., Prog. Part. Nucl. Phys. 76116 (2014) .

[6] Savajols H. and VAMOS Collaboration, Nucl. Phys. A 6541027 (1999).

[7] Pouthas J., Borderie B. and Dayras R., Nucl. Instruments Methods Phys. A 357418 (1995).

[8] Tribble R., Burch R. and Gagliardi C., Nucl. Instruments Methods Phys. A 285441 (1989).

[9] Souliotis G. A. et al., Phys. Rev. C 73024606 (2006).

[10] Gimeno-Nogues F. et al.., Nucl. Instruments Methods Phys. A 39994 (1997).

[11] Souliotis G. A. et al., Phys. Rev. C 75011601 (2007).

[12] Souliotis G. A. et al., Nucl. Instruments Methods Phys. B 2611094 (2007).

[13] Barbui M. et al.., Nucl. Instruments Methods Phys. B 265605 (2007). 
[14] Souliotis G. A. et al., Nucl. Instruments Methods Phys. B 2664692 (2008).

[15] Stein B. C. et al., AIP Conf. Proc. 700700 (2009).

[16] Swan D., Yurkon J. and Morrissey D. J., Nucl. Instruments Methods Phys. A3481994314.

[17] Davin B. et al., Nucl. Instruments Methods Phys. A 473302 (2001).

[18] Todd R., RIS-Corp (5905 Weisbrook Lane, Suite 102 Knoxville, TN 37909) 2013.

[19] Kowalski S. and Enge H., Nucl. Instruments Methods Phys. A 258407 (1987).

[20] Makino K. and Berz M., Nucl. Instruments Methods Phys. A 558346 (2006).

[21] Chapman M. B., Cammarata P. J. and Yennello S. J., Cyclotr. Annu. Reports IV91 (2014). 